\title{
LDI-Directed SIMS Imaging for Visualizing Biomolecule Distributions in Bacterial Biofilms at Micron-Scale Resolution
}

\author{
Eric J. Lanni ${ }^{1}$, Rachel Masyuko, ${ }^{2}$ Callan Driscoll, ${ }^{3}$ Joshua Shrout, ${ }^{3}$ Paul Bohn ${ }^{2}$, and Jonathan V. \\ Sweedler ${ }^{1}$ \\ 1. Department of Chemistry and Beckman Institute for Advanced Science and Technology, University of \\ Illinois at Urbana-Champaign, Urbana, IL, 61801, U.S. \\ 2. Department of Chemistry and Biochemistry and Department of Chemical and Biomolecular \\ Engineering, University of Notre Dame, Notre Dame, IN, 46556, U.S. \\ 3. Department of Civil and Environmental Engineering and Earth Sciences and Department of Biological \\ Sciences, University of Notre Dame, Notre Dame IN, 46556, U.S.
}

Many bacteria naturally grow to cover surfaces with biofilms. Biofilms are complex mixtures of surfactants, signalling molecules, and other chemical components which convey multiple advantages such as enhanced cell motility, virulence, and resistance to antibiotics. ${ }^{1}$ While significant molecular characterization has used cell suspensions in liquid media, the same bacteria can express different genes and excrete different biomolecules when growing as a biofilm, and can use quorum-sensing to transition into biofilm growth mode. ${ }^{1}$ Characterizing and spatially mapping the chemical composition of bacterial biofilms provides valuable insight into the mechanisms of formation, the function of chemical constituents, how such films respond to external influences (chemical or physical) in the environment, and how they may be controlled. We have developed methods for chemical analysis of opportunistic pathogen Pseudomonas aeruginosa biofilms by mass spectrometry imaging (MSI), allowing us to detect and visualize biomolecules including rhamnolipid surfactants which are found to be heterogeneously distributed across the surface. Laser desorption ionization (LDI) and secondary ion mass spectrometry (SIMS) are combined to take advantage of their complementary mass ranges and probe sizes. LDI is used to obtain a large-scale chemical survey of an entire sample while SIMS provides micron-scale spatial resolution at preselected regions of interest.

Biofilms of $P$. aeruginosa (ATCC strain 15692) were prepared on clean silicon wafers $1-4 \mathrm{~cm}^{2}$ in area by submersion in cell suspension for three days at $30^{\circ} \mathrm{C}$. Several strains of $P$. aeruginosa including wild type, mutant $\Delta$ las $\Delta$ rhlI (lacking rhamnolipid production and quorum-sensing capability), and isolates GM17 and GM41 were grown in this manner. Following incubation, substrates were air-dried, coated with gold or organic matrix solution, and then affixed to a sample holder for analysis. Experiments were conducted on three instruments: a MALDI-TOF/TOF MS (Ultraflextreme, Bruker Daltonics), a TOFSIMS with $\mathrm{Au}_{1}{ }^{+}$ion probe (TRIFT III, PHI), and a custom-built dual source Q-TOF MS with both UV laser and $\mathrm{C}_{60}{ }^{+}$ion probes (modified from QSTAR XL, AB SCIEX). Probe diameter on these instruments was typically $50 \mu \mathrm{m}, 10 \mu \mathrm{m}$, and $1 \mu \mathrm{m}$ respectively. Spectra were typically acquired over $m / z$ 20-2000 in positive ion mode.

Several chemical treatments were compared for performance by evaluating signal enhancement of rhamnolipid standards. Compared with conventional organic MALDI matrices, gold sputter coating for metal-assisted (MetA) LDI was found to yield superior overall performance: comparable signal strength, less chemical interference below $\mathrm{m} / \mathrm{z}$ 1000, no significant reduction of lateral imaging resolution as with crystallizing matrices, improved sample conductivity to avoid charging effects, and Au cluster ions convenient for mass calibration. Gold coating thickness was optimized at 1-2 nm. For SIMS analysis 
with $\mathrm{Au}_{1}^{+}$microprobe, this preparation was found to increase rhamnolipid signals up to 10-fold. With a $\mathrm{C}_{60}{ }^{+}$microprobe the same ions were found to be almost completely suppressed by gold coating.

For a large-scale survey of biofilm samples, MetA-LDI was performed over the entire substrate at large $(>300 \mu \mathrm{m})$ raster size, leaving substantial unperturbed surface between probe points for subsequent SIMS analysis. MS images of wild type $P$. aeruginosa (see Fig. 1) reveal spatial heterogeneity for many dozen detected ions, including salt adducts of several rhamnolipids such as alpha-L-rhamnopyranosylalpha-L-rhamnopyranosyl-beta-hydroxydecanoyl-beta-hydroxydecanoate (Rha-Rha-C10-C10, $\mathrm{m} / z$ 673), confirmed by in situ tandem MS. In contrast with wild type, no rhamnolipids were detected on mutant $\Delta$ las $\Delta r h l I$ biofilms by MetA-LDI although other unidentified ions were detected in greater abundance and warrant further characterization. SIMS analysis reveals chemical heterogeneity at $<20 \mu \mathrm{m}$ size scales (see Fig. 2) and similar rhamnolipid-related ions are detected in profiling experiments conducted at sample regions where LDI has detected high rhamnolipid abundance. Present and future work is focused on identification of unknown ions and precise specification of SIMS analysis regions using the LDI chemical map.

[1] Costerton, J. W. et al., Ann Rev Microbio 49, p. 711.

[2] The authors acknowledge funding from the Department of Energy Office of Science (BER) through grant DE-SC0006642. Bacterial strain provision from Dr. Jennifer Morrel-Falvey and Sarah Melton at ORNL is gratefully acknowledged.
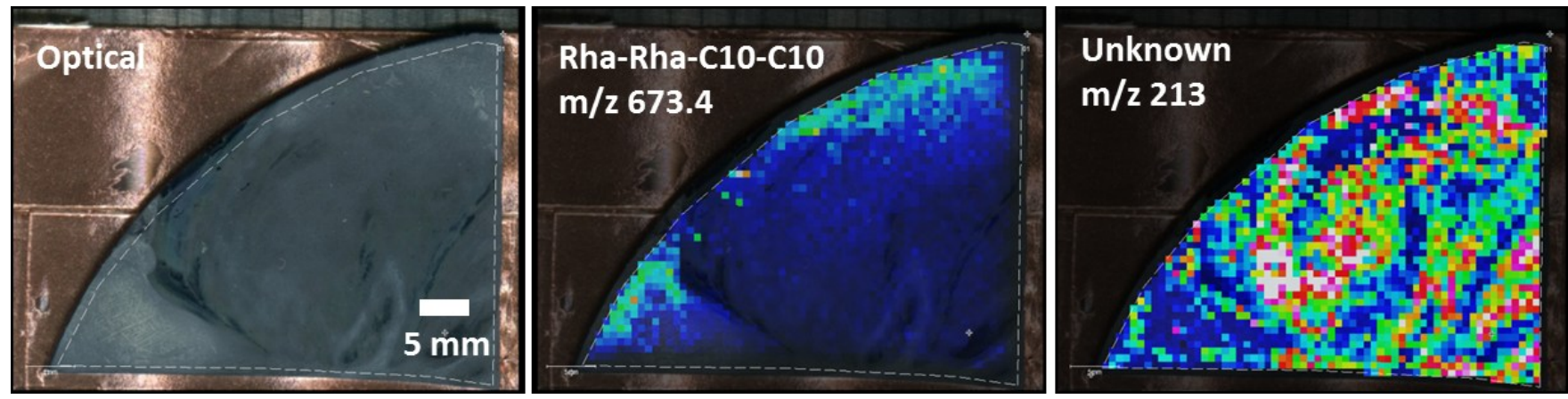

Figure 1. MetA-LDI reveals spatiochemical heterogeneity across $P$. aeruginosa wild type biofilm grown on Si wafer. A rhamnolipid (Rha-Rha-C10-C10) is concentrated at the edge of the substrate while unidentified ion $\mathrm{m} / \mathrm{z} 213$ appears heterogeneously distributed throughout.
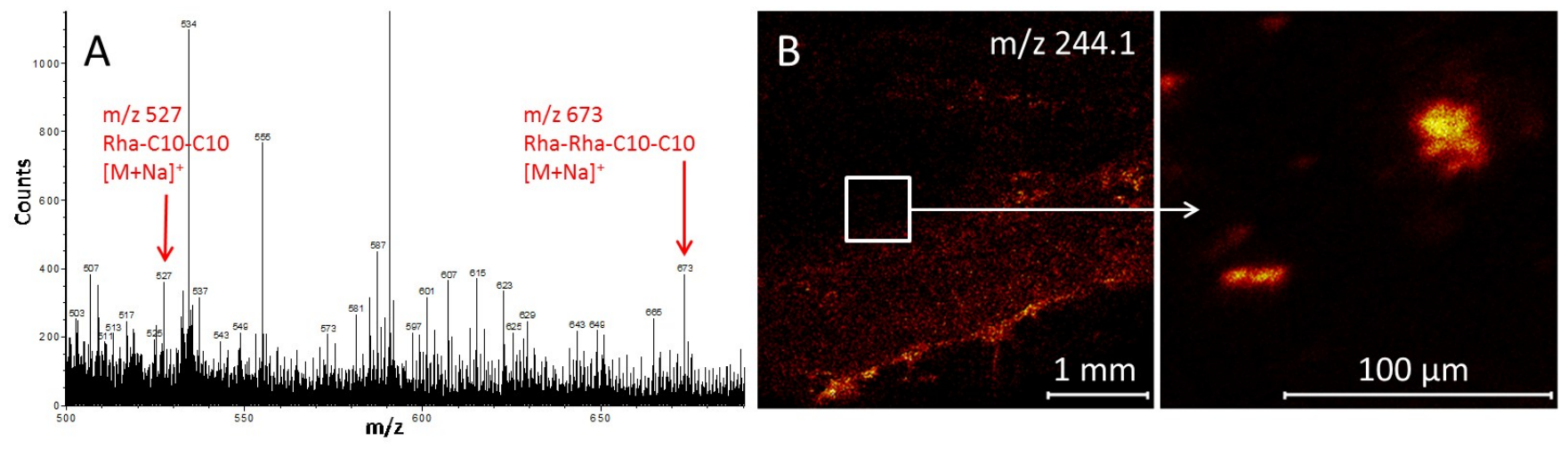

Figure 2. MetA SIMS analysis of wild type $P$. aeruginosa biofilm A) detects intact rhamnolipid-related ions similar to those observed by MetA-LDI and B) resolves micron-scale chemical heterogeneity. 